\title{
Development of the Bodily Self: Effects of Visuomotor Synchrony and Visual Appearance on Virtual Embodiment in Children and Adults
}

\author{
Marieke L. Weijs ${ }^{1}$, Elle Macartney ${ }^{1}$, Moritz M. Daum ${ }^{1,2}$, Bigna Lenggenhager ${ }^{1}$
}

${ }^{1}$ Department of Psychology, University of Zurich, Zurich, Switzerland

${ }^{2}$ Jacobs Center for Productive Youth Development, Zurich, Switzerland

Corresponding author: Marieke Weijs, Department of Psychology, University of Zurich Binzmühlestrasse 14, Box 9, marieke.weijs@uzh.ch

Conflict of interest statement: The authors report no conflict of interest.

Acknowledgements: This research was supported by the Swiss National Science Foundation (grant number: 170511). We thank Marte Roel Lesur for help with the virtual reality setup, Sonia Lyn and Luisa Casanova for assistance with data collection, and Melanie Schwaniger for help with preprocessing of the skin conductance data. Thanks to the participants and parents for taking part in this study.

Data availability statement: Anonymized data and code are available from https://osf.io/ef2bz/ 


\section{Research Highlights}

- We assessed children's and adult's subjective and physiological responses to a firstperson perspective full body illusion in virtual reality, manipulating appearance and visuomotor synchrony.

- Children and adults reported similar subjective ownership, which was reduced with increasing asynchrony and less human visual similarity, but not reflected in objective measures.

- Overall, children experienced more agency than adults, which was also less affected by increasing asynchrony or avatar appearance than in adults.

- The findings demonstrate that children easily embody an avatar, and enjoy the experience more than adults, which highlights the great potential of embodied virtual reality. 


\begin{abstract}
The sense of a bodily self is thought to depend on adaptive weighting and integration of bodily afferents and prior beliefs. Evidence from studies using paradigms such as the rubber hand illusion and full body illusion suggests changes in the integration of visuotactile bodily signals throughout childhood. Here, we extended this line of research by assessing how bottom-up visuomotor synchrony and expectancy, modulated by visual appearance of virtual avatars, contribute to embodiment in children. We compared responses to a first-person perspective virtual full body illusion from 8-12-year-old children and adults, while manipulating synchrony of the avatar's movements (synchronous, $0.5 \mathrm{~s}, 1 \mathrm{~s}$ delay compared to the participant's movements), and appearance of the avatar (human or skeleton). We measured embodiment with both subjective questionnaires and objective skin conductance responses to virtual threat. Results showed that children experienced ownership for the virtual avatar in a similar way to adults, which was reduced with increasing asynchrony, and for the skeleton avatar as compared to the human avatar. This modulation of ownership was not reflected in the skin conductance responses, which were equally high in all experimental conditions and only showed a modulation of repetition by age. Contrastingly, in children, the subjective experience of agency was less affected by the dampening effects of visuomotor asynchrony or reduced human likeness, and overall higher. These findings suggest that children can easily embody a virtual avatar, but that different aspects of embodiment develop at different rates, which could have important implications for applications of embodied virtual reality.
\end{abstract}

Key words: bodily self, multisensory integration, virtual reality, body ownership, agency, full body illusion 


\section{Development of the Bodily Self: Effects of Visuomotor Synchrony and Visual}

\section{Appearance on Virtual Embodiment in Children and Adults}

The feeling of having a body (i.e. sense of ownership) and sensing control over it (i.e. sense of agency), are typically not questioned in daily life. Still, these core aspects of the sense of a bodily self result from a constant and complex weighting and integration of sensory information from in- and outside our body with longer-term priors about our body (Apps \& Tsakiris, 2014). The developing body in childhood provides a unique possibility to study underlying mechanisms, as it is characterized by rapid changes of shape, size, and functionality of the physical body, resulting in the need to constantly adjust such multisensory integration processes (Adolph \& Hoch, 2019; Thelen, 1992). In this study we investigate differences between children and adults in experiences of body ownership and agency during experimental manipulations of embodiment.

In adults, various experimental paradigms have been developed to investigate the contribution of bottom-up integration and top-down priors to different aspects of the sense of a bodily self. A famous example is the rubber hand illusion (Botvinick \& Cohen, 1998), where matching visuotactile cues from a rubber hand in front of the participant and from the unseen real hand can induce the feeling of ownership of this rubber hand. Such illusion is reflected in subjective reports (Longo et al., 2008), behavioral proprioceptive drift responses (Tsakiris \& Haggard, 2005), and in physiological changes, such as an increased skin conductance response (SCR) to a threat to the rubber hand (Armel \& Ramachandran, 2003, but see Kokkinara \& Slater, 2014 for non-replication). These manipulations of multisensory bodily information can be extended to the full body, which elicits the feeling of body ownership of a completely foreign body, and is also accompanied by threat-evoked SCR (Petkova \& Ehrsson, 2008). Classically, the temporally synchronous but conflicting multisensory information to induce such temporary alterations in the bodily self is provided 
by visuotactile stimulation (Botvinick \& Cohen, 1998), but similar effects have been found in the visuomotor domain (Kalckert \& Ehrsson, 2012). This is especially important for embodied virtual reality applications, where the virtual avatar is typically seen from a firstperson perspective and illusory body ownership is established by matching of the avatar's and the user's tracked body movements (Maselli \& Slater, 2013; Slater et al., 2010).

Multisensory integration has received much attention in developmental research (Bahrick \& Lickliter, 2000; Bahrick \& Watson, 1985; Zmyj et al., 2011), and is seen as a fundamental aspect for the development of a bodily self (Bahrick, 2013; Riva, 2018; Rochat, 2003). Therefore, investigating how multisensory integration directly underlies subjective feelings of agency and body ownership might deepen our understanding of how the sense of bodily self develops. Body morphology and functionality as well related structural and functional brain mechanisms (Mills et al., 2016; Power et al., 2010) change at a fast rate during childhood. Such rapid changes in the body bring about new body-environment relations, and require a constant adjustment of multisensory integration (Burr \& Gori, 2011; King et al., 2010; Nardini et al., 2013), and locomotor skills to be able to successfully interact with the environment experienced from a novel perspective (Adolph \& Hoch, 2019). Already in infancy, a sensitivity for multimodal contingencies between the own body and the outside world has been observed, for example in visuoproprioceptive or visuotactile displays (Bahrick \& Watson, 1985; Zmyj et al., 2011). Furthermore, it has been argued that awareness of multimodal synchrony drives the ability to distinguish between 'me' and 'not me', which is essential for the developing sense of self (Bahrick, 2013), which is the mechanism driving above-described bodily illusions in adults. What remains under-investigated is how the integration of different multisensory signals contributes to maintenance and plasticity of a sense of bodily self in childhood. Recently, the role of visuotactile signals for the sense of limb ownership in children, using the rubber hand illusion has been addressed. Studies using 
the rubber hand illusion (Cowie et al 2013, 2016), a nonvisual version of the rubber hand illusion (Nava et al. 2017), and a video-based full body illusion where the body was seen from a third-person perspective (Cowie et al., 2017) reported differences in the sense of ownership as measured in explicit and implicit behavioral measures between children and adults.

Yet, while these studies have focused on the role of visuotactile cues on the bodily self, it has been suggested that bodily action might play a more important role in the formation and maintenance of a coherent sense of bodily self both in adults and throughout development (Bahrick \& Watson, 1985; Kokkinara \& Slater, 2014; Nava et al., 2018; Tsakiris et al., 2006). In adults, it has for example been shown that when an avatar moves in synchrony with the participant's movements, mismatching visuotactile cues less strongly reduce embodiment, suggesting that visuomotor synchrony is the driving force behind the illusion of ownership (Kokkinara \& Slater, 2014; Roel Lesur, Weijs, et al., 2020). Here, we thus investigated how visuomotor coherence, with different delays between movements of the participant and avatar, affects body ownership and agency in children as compared to adults in an immersive first-person perspective full body illusion in virtual reality.

Importantly, it is not only bottom-up sensorimotor coherencies, but also visual appearance of artificial bodies or body parts, that have been shown to crucially determine the strength of experimentally induced alterations of body ownership. Traditionally, human-like characteristics in terms of topology and anatomy have been considered a baseline requirement for such illusions. For example, participants did not experience ownership over a rectangular block in either the rubber hand illusion (Tsakiris \& Haggard, 2005) or the full body illusion (Lenggenhager et al., 2007). Yet, it has been argued that, especially when visuomotor head movement contingency is present, such restrictions are less present (Roel Lesur, Aicher, et al., 2020; Roel Lesur et al., 2018). Still, it is generally agreed that with an 
increasing human resemblance, there is a strengthening effect of the feeling of illusory ownership of the fake hand or virtual body (Haans et al., 2008; Maselli \& Slater, 2013), possibly due to top-down expectancies of visual appearance of the own body. The use of a virtual reality setup provides an excellent opportunity to flexibly manipulate the visual appearance of virtual avatars, which we used to assess how human likeness affects embodiment.

To test our two main hypotheses about how children and adults are differently affected by avatar appearance and visuomotor coherence, we manipulated in a withinsubjects design the visual appearance of the virtual avatar, so it resembled either a genderneutral human or a skeleton, and visuomotor synchrony by showing the avatar's movements either in synchrony with the participant's movements or with a 0.5 to $1 \mathrm{~s}$ delay. Comparably to previous related studies (Cowie et al., 2016, 2017), we here focused on children between 8 and 12 years old, the lower limit being defined by the safe use of the head-mounted display (HMD) for the duration of our study and the upper limit being defined as start of puberty (Spear, 2000). Embodiment was assessed both explicitly, with an embodiment questionnaire including items on the sense of ownership and agency, and implicitly, by measuring SCRs to a virtual threat. As children have previously shown to rely more on visual cues (visual capture), and to be less sensitive to visuotactile delay than adults (Cowie et al., 2016, 2017), we expected that ownership, as measured both implicitly and explicitly, and agency would be stronger in children and less modulated by visuomotor delay as compared to adults. Due to potentially stronger reliance on body memory with age, we expected adults to be more affected by top down constrains than children. To test this, we used a human-like versus a skeleton avatar, and expected the latter in line with previous literature to induce less ownership and agency in adults, while we did expect a less pronounced difference between the avatars in children. 
Next to these main hypotheses on the difference between adults and children, we aimed at investigating how the children's age affects the sense of ownership and agency in the mentioned conditions, in a more explorative manner due to limited sample size. Previous research using visuotactile body illusions has shown that there are developmental differences in responses to visuotactile body illusions. Specifically, there seems to be a developmental dissociation between subjective responses and proprioceptive drift, both in visual (Cowie et al., 2013, 2016), and nonvisual (Nava et al., 2017) versions of the rubber hand illusion. Children between 4 and 10 years old subjectively reported ownership of the rubber hand after synchronous visuotactile stimulation, but did not show adult-like proprioceptive drift responses (i.e. a stronger drift after synchronous compared to asynchronous stimulation) until the age of 10 years (Cowie et al., 2013, 2016). Taken together, these studies suggest that binding of visuotactile signals, which might drive body ownership, becomes more adult-like around the age of 10 years, and that visual capture mainly drives the sense of body ownership before that age (Cowie et al., 2016, 2017). Similar effects might occur with regards to visuomotor stimulation, and we used a median split to divide the children in two groups, below and above 10.5 years old to compare younger and older children in exploratory analyses.

\section{Methods}

\section{Participants}

Thirty-two children between 8 and 12 years old (Mean age: 10.2 years, $S D=1.1 ; 23$ female), and 33 adults (Mean age: 23.1 years, $S D=2.8 ; 25$ female) participated in the study. All participants were healthy and reported normal or corrected-to-normal vision. The study was approved by the Ethics Committee of the Faculty of Arts and Social Sciences at the University of Zurich (Approval number 17.12.15), and was conducted in accordance with the ethical standards of the Declaration of Helsinki. All participants (and their parents for the 
children group) provided informed consent. Children were recruited via a database of parents who volunteered to participate in developmental studies, and received a gift worth approximately $5 \mathrm{CHF}$ as compensation for participation. Adults were recruited via university mailing lists and advertisements, and received course credit or $15 \mathrm{CHF}$ in exchange for participation.

\section{Design}

A $2 \times 3$ within-subjects design was used in this study, manipulating avatar appearance (human or skeleton) and visuomotor synchrony (synchronous, $0.5 \mathrm{~s}$ delay, $1 \mathrm{~s}$ delay). The six conditions were presented in counterbalanced order. We measured physiological responses to threat in each condition, and participants completed a 6-item embodiment questionnaire after each condition. The order of conditions was counterbalanced across participants.

\section{Experimental Setup}

The experiment took place in a room set up with a VIVE Pro system with two base stations (HTC, Taipei, Taiwan). Four additional VIVE trackers and a controller were used to implement full body tracking. The trackers were placed on the ankles and wrists with Velcro bands, and the controller was placed in the center of the hips. The virtual environment, and experimental procedure were created and implemented in Unity (Version 2018.2, Unity Technologies, San Francisco, USA). The virtual room was modeled after the physical room, and matched in terms of size and furnishings. An additional virtual mirror was placed above the reclining chair in the virtual room, allowing participants to view their full body (see Figure 1A). This mirror was not present in the physical room. The human avatar was created in MakeHuman (www.makehumancommunity.org) and modeled after a 10-year-old child with neutral gender, and the skeleton avatar was downloaded from the Unity Asset Store (see Figure 1A). The avatar's movements were implemented through an inverse kinematics solution, using the Final IK package in Unity (www.root-motion.com/final-ik.html). 
Additional adjustments were made in Unity to provide a plausible body structure while the avatar was in a lying position on the reclining chair. The avatars were scaled for each participant individually, based on the distance between the ankle trackers and HMD while standing to ensure a matching body size between the participant and the avatars.

Physiological signals were recorded with a $1000 \mathrm{~Hz}$ sampling rate using a Biopac MP150 system with EDA100C and ECG100C amplifiers (Goleta, USA). Data was stored in Acqknowledge software (Version 4.1, Goleta, USA). Triggers marking the start, virtual threat, and end of each experimental condition were sent from Unity to the recording computer. ECG recordings were not used for the subsequent analyses.

\section{Procedure and Measures}

The experiment started with a verbal explanation of the procedure. The children group was shown a printed version of the Visual Analogue Scale (VAS) scale with two unrelated statements, to demonstrate how the scale works. Participants were then fitted with the ECG and EDA electrodes, the trackers, and HMD. Afterwards, participants lay down on a reclining chair, to ensure that participants could see the entirety of the virtual body. Before the actual experiment, a practice trial was included as to show how to use the HMD to answer questions on a VAS scale with the same two example statements as shown in the printed version before.

The experiment started with an embodiment induction phase of $40 \mathrm{~s}$ in which participants followed verbal instructions presented on the earphones of the HMD (see Figure 1 for the full procedure). Participants were instructed to lift their arms and legs one by one and put them back down, while either looking down at the virtual body, or looking up in a virtual mirror. This was placed above the reclining chair as to show the reflection of the full avatar. A second phase consisted of an interactive game that lasted $90 \mathrm{~s}$, to maximally engage participants with the virtual body and ensure that they would actively use it (Figure 1B). 
Participants were instructed to reach for virtual cubes with their arms and legs. A total of ten cubes appeared one by one in locations above the body. A successful interaction, caused by a collision of a tracker with the cube, prompted a change in color of the cube from white to yellow. Each cube disappeared after $5 \mathrm{~s}$, regardless of a successful interaction.

Finally, a virtual threat was presented to measure participants' emotional reaction to it using SCRs. This threat was a diamond shaped virtual object falling from the ceiling, and moving through the virtual body (Figure 1C). This object was chosen based on a trade-off between a risk of injuring the virtual body, and a fear of the threat which may have been triggered by the more classically used knife in studies testing adult participants (e.g. Petkova \& Ehrsson, 2008). After the threat had moved through the avatar, the body remained visible for another $10 \mathrm{~s}$ in which no prompts for movements were show. This avoided contamination of the threat-evoked SCR with potential movement artefacts or responses triggered by a change in the visual stimulation, adding up to an embodiment phase of $150 \mathrm{~s}$ in total.

After this point, an embodiment questionnaire was presented on the HMD that could be answered by selecting a point on a VAS scale using head movements (Figure 1D). Subjective feelings of embodiment, overall enjoyment, and strangeness were assessed with a 6-item embodiment questionnaire (Table 1), which was compiled using statements from previous experiments using the full body illusion in children and adults (Cowie et al., 2017; Lenggenhager et al., 2007), and the rubber hand illusion in children (Cowie et al., 2016). A control statement was included in the questionnaire to assess overall tendency to agree. Participants indicated agreement with each statement on a VAS scale from "Completely disagree" to "Completely agree". Ratings were coded as a value between 0 and 1 , where 0 indicated complete disagreement and 1 complete agreement. The visuomotor stimulation and questionnaire were repeated six times in total, to cover all experimental conditions. The experiment lasted approximately 25 minutes in total. 


\section{Data Processing and Statistical Analyses}

Preprocessing of the questionnaire data and statistical analyses were performed in $\mathrm{R}$ version 3.6.1 (R Core Team, 2018). The alpha level was set at 0.05. Responses to the questionnaire generally demonstrated a non-normal distribution, therefore a non-parametric approach was chosen. To assess the effects of avatar (2 levels, human and skeleton), synchrony ( 3 levels, synchronous, $0.5 \mathrm{~s}$ delay, $1 \mathrm{~s}$ delay), and group ( 2 levels, children, and adults) aligned rank transformed repeated measures ANOVAs were used. These are implemented in the ARTool (Kay \& Wobbrock, 2020), and apply linear mixed models to the aligned rank transformed data. Post-hoc comparisons for effects and contrasts of interest were calculated using Wilcoxon Signed-Rank tests with Bonferroni corrections for multiple comparisons. Plots were created in $\mathrm{R}$ with the ggplot 2 package using a modified raincloud plots script (Allen et al., 2019).

The same steps were followed for exploratory analyses within the two children groups. To be able to compare younger and older children with the limited sample size, we performed a median split on the age ( $M d n=125.5$ months). This resulted in a group of $<10.5$ years and $>10.5$ years of each 16 children.

SCR data processing and analysis. Nine adults and three children had to be excluded from the analysis of skin conductance data, due to measurement errors $(N=4)$, and missing triggers $(N=10)$. For all other participants electrodermal activity data were analyzed in Acqknowledge software (Version 4.1, Goleta, USA). The amplitude of each response was defined as the through-to-peak value in the 6-14 second period after the trigger. A trigger was sent the moment the object started falling from the ceiling. However, as participants generally did not see the object in the first seconds, only SCRs in the specified time period were considered. Only amplitudes over $0.02 \mu \mathrm{S}$ were considered, and absent responses were registered as 0 . Individual Z-scores were calculated, to account for the large inter-individual 
variance in SCRs (Dawson et al., 2000). The SCRs were analyzed with a linear mixed model, to be able to include participants for which responses in some conditions were missing, and to address the potential order in the analyses. The final model included participant as a random effect, and avatar, synchrony, age group, order, and their interactions, as fixed effects.

\section{Results}

Firstly, we assessed whether there were any effects of groups or experimental manipulations on the responses to the control statement to test for suggestibility. Overall, participant's tendency to agree with the control statement was low $(M d n=0.07$, $I Q R=0.03-0.21)$. The repeated measures ART ANOVA did not show any significant effects of avatar $\left(F(1,312)=0.89, p=.35, \eta_{p}^{2}=0.003\right)$, synchrony $(F(2,312)=0.73$, $\left.p=.48, \eta_{p}^{2}=0.004\right)$, group $\left(F(1,61)=1.96, p=.17, \eta_{p}^{2}=0.03\right)$, or the two-way and threeway interactions (all $F \mathrm{~s}<1.92$, all $p \mathrm{~s}>.15, \eta_{p s}^{2}<0.01$ ) on responses to the control statement. Therefore, we assumed that the tendency to agree would not be affected by the experimental manipulations or age group, and we did not apply any adjustments to the other questionnaire ratings.

\section{Subjective Experience of Ownership and Agency}

With regards to the experience of ownership, there was a main effect of avatar, $\left.F(1,312)=24.77, p<.001, \eta^{2} p=0.07\right)$, and of synchrony $(F(2,312)=9.12, p<.001$, $\eta_{p}^{2}=0.06$; see Figure 2A for responses in all conditions). Participants reported overall more ownership for the human $(M d n=0.57, I Q R=0.35-0.76)$ than for the skeleton avatar $(M d n=$ $0.45, I Q R=0.23-0.61)$. Post-hoc comparisons for synchrony demonstrated that ownership was highest in the synchronous condition $(M d n=0.57, I Q R=0.38-0.77)$, and significantly reduced when the avatar's and participant's movements were asynchronous for both the $0.5 \mathrm{~s}$ $(M d n=0.49, I Q R=0.28-0.67 ; Z=-4.22, p<.001, r=-0.27)$, and the $1 \mathrm{~s}$ delay condition 
$(M d n=0.44, I Q R=0.24-0.64 ; Z=-4.46, p<.001, r=-0.28)$. Ownership ratings did not differ significantly between both asynchronous conditions $(Z=-0.39, p=.70, r=-0.02)$. There was no significant effect of age group, or any of the two-way or the three-way interaction (all $F \mathrm{~s}<1.36$, all ps $>.26, \eta_{p s}^{2}<0.009$ ).

Regarding the experience of agency, there was a main effect of avatar $\left(F(1,312)=8.64, p=.004, \eta^{2} p=0.03\right)$, of synchrony $(F(2,312)=48.99, p<.001$ $\left.\eta^{2}{ }_{p}=0.24\right)$, and of age group $\left(F(1,61)=11.73, p=.001, \eta^{2} p=0.16\right.$; see Figure $2 \mathrm{~B}$ for responses in all conditions). In addition to the significant main effects for all factors, there was a significant avatar and age group interaction $\left(F(1,312)=7.62, p=.006, \eta^{2} p=0.02\right)$. Post-hoc comparisons showed that agency for the human $(M d n=0.73, I Q R=0.42-0.88)$ and skeleton $(M d n=0.70, I Q R=0.48-0.88)$ did not differ significantly in the children group $(Z=-0.99, p=.32, r=-0.07)$. On the other hand, adults experienced more agency for the human avatar $(M d n=0.60, I Q R=0.41-0.78)$ than for the skeleton $(M d n=0.51, I Q R=0.24$ $-0.65 ; Z=-3.77, p<.001, r=-0.27)$. For both the human $(Z=-2.32, p=.041, r=-0.17)$ and the skeleton avatar $(Z=-4.61, p<.001, r=-0.33)$, agency was significantly higher in the children group than in the adult group. Finally, there was a significant interaction of synchrony and age group $\left(F(2,312)=4.30, p=.014, \eta^{2} p=0.03\right)$. Post-hoc comparisons for the contrasts of interest showed that children experienced significantly more agency in the synchronous $(M d n=0.87, I Q R=0.64-0.96)$ than in the $0.5 \mathrm{~s}(M d n=0.67, I Q R=0.45$ $0.85)$ condition $(Z=-3.09, p=.018, r=-0.28)$. and in the $1 \mathrm{~s}$ condition $(M d n=0.66, I Q R=$ $0.39-0.8 ; Z=-4.16, p<.001, r=-0.37)$, but there was no significant difference between the two asynchronous conditions in the children group $(Z=-0.93, p=1.00, r=-0.08)$. In the adult group, agency significantly reduced from the synchronous $(M d n=0.70, I Q R=0.58$ $0.88)$ to the $0.5 \mathrm{~s}$ asynchronous condition $(M d n=0.54, I Q R=0.30-0.72 ; Z=-5.00, p<$ $.001, r=-0.44)$, and from the synchronous to the $1 \mathrm{~s}$ asynchronous condition $(M d n=0.32$, 
$I Q R=0.20-0.56 ; Z=-6.11, p<.001, r=-0.54)$. Agency was significantly lower in the $1 \mathrm{~s}$ than 0.5 s condition $(Z=-4.35, p<.001, r=-0.38)$. While agency did not differ significantly between children and adults in the synchronous condition $(Z=2.44, p=.131, r=-0.21)$ or in the 0.5 s condition $(Z=-2.72, p=.059, r=-0.24)$, in the $1 \mathrm{~s}$ condition it was significantly higher for children than for adults $(Z=-4.34, p<.001, r=-0.38)$.

\section{Subjective and Objective Responses to Threat}

The subjective experience of the threat, the response to the question whether the threat could actually hurt, did not show any significant effects of experimental manipulations and age group, or their interactions $(M d n=0.41, I Q R=0.19-0.73$; all $F \mathrm{~s}<3.51, p \mathrm{~s}>.07$, $\eta_{p s}^{2}<0.05 ;$ see Figure 3A).

Overall, objective responses were present in most of the trials, showing that the threat effectively elicited an SCR. The number of responses was higher in children $(M d n=6$, $I Q R=6-6)$ than in adults $(Z=-3.23, p=.001, r=-0.44)$, but also in adults, a response was present in most of the trials $(M d n=5, I Q R=4-6)$. The model explaining SCR responses by group, synchrony, avatar and order $\left(R_{m}^{2}=0.24\right)$ showed a significant effect of order $(F(5,239)=5.95, p<.001)$, and a significant interaction of age group and order $(F(5,239)=10.55, p=.019)$. All other effects were not significant (all $\left.F_{\mathrm{s}}<2.78, p \mathrm{~s}>.16\right)$. While adults demonstrated a clear reduction in the SCR over repetitions, children did not show this reduction (significant linear effect of order in the adult group: $b=-8.00, S E=1.55$, $t(203)=-5.16, p<.0001, d=-9.17$, but not in the children group: $b=-1.70, S E=1.47$, $t(203)=-1.15, p=.25, d=-1.94$, see Figure 3B).

\section{Affective Responses to the Virtual Reality Experience}

Finally, we assessed whether there were differences in subjective appraisal of the illusion in terms of strangeness and enjoyment between conditions and groups. For strangeness, there was a significant main effect of avatar $(F(1,312)=13.27, p<.001$, 
$\left.\eta_{p}^{2}=0.04\right)$ and of synchrony $\left(F(2,312)=4.59, p=.012, \eta_{p}^{2}=0.03\right)$, as well as a significant interaction between these two factors $\left(F(2,312)=3.73, p=.025, \eta^{2} p=0.02\right)$. All other effects were not significant (all $F \mathrm{~s}<2.28, p \mathrm{~s}>.10, \eta_{p s}^{2}<0.01$ ). Post-hoc comparisons showed that strangeness for the human avatar did not differ significantly between the synchronous $(M d n=0.51, I Q R=0.30-0.67)$ and $0.5 \mathrm{~s}$ delay condition $(M d n=0.47, I Q R=$ $0.27-0.60 ; Z=-1.05, p=1.00, r=-0.09)$, or between the synchronous and $1 \mathrm{~s}(M d n=0.59$, $I Q R=0.37-0.75)$ condition $(Z=-2.47, p=.12, r=-0.22)$, it was significantly stronger for the $1 \mathrm{~s}$ than $0.5 \mathrm{~s}$ condition $(Z=-3.18, p=.013, r=-0.28)$. For the skeleton, there were no significant differences between the synchronous $(M d n=0.51, I Q R=0.35-0.70)$ and either of the asynchronous conditions $(0.5 \mathrm{~s} M d n=0.61, I Q R=0.39-0.79 ; 1 \mathrm{~s} M d n=0.59, I Q R=$ $0.37-0.75$; all $Z \mathrm{~s}>-2.27, p \mathrm{~s}>.21, r \mathrm{~s}>-0.09)$. Comparisons of strangeness between the human and skeleton at each level of synchrony demonstrated that strangeness did not differ in either the synchronous $(Z=-1.30, p=1.00, r=-0.12)$ or $1 \mathrm{~s}$ delay condition $(Z=-1.56, p=$ $1.00, r=-0.14)$, but it was significantly higher for the skeleton than the human in the $0.5 \mathrm{~s}$ condition $(Z=-3.93, p<.001, r=-0.35)$.

The final question concerned enjoyment of the experience in each experimental condition. Here, there were significant main effect for avatar $(F(1,312)=25.04, p<.001$, $\left.\eta_{p}^{2}=0.07\right)$, synchrony $\left(F(2,312)=11.59, p<.001, \eta_{p}^{2}=0.07\right)$, and age group $(F(1,61)=$ 13.72, $\left.p<.001, \eta^{2} p=0.18\right)$. Additionally, there was a significant interaction of avatar and age group $\left(F(1,312)=19.84, p<.001, \eta^{2} p=0.06\right)$. For children, there was overall no difference in enjoyment between the human $(M d n=0.94, I Q R=0.75-0.98)$ and skeleton avatar $(M d n$ $=0.93, I Q R=0.73-0.98 ; Z=-1.15, p=.25, r=-0.08)$, but adults enjoyed the experience more in the human $(M d n=0.75, I Q R=0.64-0.89)$ than skeleton avatar $(M d n=0.68, I Q R=$ $0.51-0.79 ; Z=-3.76, p<.001, r=-0.27)$. Overall, children enjoyed the experience more than adults in both the human $(Z=-4.59, p<.001, r=-0.33)$, and the skeleton condition $(Z$ 
$=-5.75, p<.001, r=-0.42)$. Finally, there was also a significant interaction of synchrony and age group $\left(F(2,312)=4.42, p=.013, \eta^{2} p=0.03\right)$, showing that there was no significant difference in enjoyment for the children between the synchronous $(M d n=0.95, I Q R=0.76$ $0.98), 0.5$ s delay condition $(M d n=0.90, I Q R=0.76-0.99)$, and $1 \mathrm{~s}$ delay condition $(M d n=$ $0.91, I Q R=0.73-0.97 ;$ all $\mathrm{Zs}>-2.36, p \mathrm{~s}>.16, r \mathrm{~s}>-0.21)$. For adults, enjoyment was significantly lower in the $1 \mathrm{~s}$ condition $(M d n=0.68, I Q R=0.52-0.79)$ as compared to both the synchronous condition $(M d n=0.72, I Q R=0.65-0.87 ; Z=-3.94, p<.001, r=-0.35)$, and the 0.5 s delay condition $(M d n=0.73, I Q R=0.59-0.87 ; Z=-2.84, p=.041, r=-0.25)$, but did not differ significantly between the synchronous and $0.5 \mathrm{~s}$ condition $(Z=-2.24, p=.23$, $r=-0.20)$. In all of the synchrony conditions, children reported higher enjoyment than adults (synchronous vs $0.5 \mathrm{~s}: Z=-4.16, p=<.001, r=-0.37$; synchronous vs $1 \mathrm{~s}: Z=-4.11$, $p=<.001, r=-0.37 ; 0.5 \mathrm{~s}$ vs. $1 \mathrm{~s}: Z=-4.52, p=<.001, r=-0.40)$. The other interaction effects were not significant (all $F \mathrm{~s}<1.13, p \mathrm{~s}>.32, \eta_{p s}^{2}<0.007$ ).

\section{Exploratory Analyses of Age Effects within the Children Group}

In exploratory analyses, we further investigated whether there was a difference between younger ( $<10.5$ years old) and older children $(>10.5$ years old) in responses to the questionnaire. For this reason, we added an additional factor with two levels, namely children's age group, and analyzed exclusively the data from the children group. Here, we only focus on effects that involve children's age group (i.e. main effect of children's age group, and children's age group interaction with avatar and synchrony). Within the children group, there were no effects of children's age group on responses to the control statement (all $\left.F_{\mathrm{s}}<1.98, p \mathrm{~s}>.14, \eta_{p s}^{2}<0.03\right)$, thus the tendency to agree was comparable across younger and older children. Both for ownership (all $F \mathrm{~s}<3.82, p \mathrm{~s}>.06, \eta_{p s}^{2}<0.11$ ), and agency (all $\left.F_{\mathrm{s}}<1.31, p \mathrm{~s}>.25, \eta_{p s}^{2}<0.009\right)$, there were no children's age group-related effects. For strangeness there was a significant interaction of synchrony and children's age group 
$\left(F(2,145)=3.53, p=.032, \eta^{2} p=0.05\right)$. This effect was driven by higher strangeness in the 1 s delay condition $(M d n=0.63, I Q R=0.42-0.82)$ than in the $0.5 \mathrm{~s}$ delay condition $(M d n=0.50, I Q R=0.12-0.68)$ in the older children group. However, the difference did not remain significant after Bonferroni correction $(Z=-2.68, p=.066, r=-0.47)$. All other comparisons were also not significant (all $\mathrm{Zs}<-2.36, p \mathrm{~s}>.17$ ). Finally, there were also no children's age group-related effects for enjoyment (all $F_{\mathrm{S}}<2.42, p \mathrm{~s}>.13, \eta_{p s}^{2}<0.08$ ), and for subjective threat experience (all $F_{\mathrm{s}}<1.10, p \mathrm{~s}>.33, \eta_{p s}^{2}<0.018$ ).

\section{Discussion}

In this study, we set out to investigate the difference between children's and adult's subjective and objective responses to a VR-based visuomotor first-person perspective full body illusion. We measured whether they show differential sensitivity to prior expectancy as manipulated by physical appearance of the virtual avatar, and to multisensory coherency as manipulated by different visuomotor delays. Overall, we found predicted effects in adults and a dissociation between children and adults in subjective experiences of agency and ownership. In adults, we found a modulatory effect of both top-down and bottom-up manipulations on the subjective sense of ownership and agency, which was however not supported by implicit measures. On the other hand, children generally showed a stronger sense of agency than adults, both with increasing temporal mismatches and also for the skeleton avatar, but no differences in ownership responses, which suggests a differential development of the sense of ownership and agency. This finding adds a developmental perspective to the ongoing research on the relation and dissociation between agency and body ownership (Pyasik et al., 2018; Seghezzi et al., 2019). Based on previous multisensory stimulation paradigms in adults, the sense of bodily agency (i.e. the sense that one is in control of their own body) and ownership have been suggested to be closely related, but they can also be individually modulated (Cioffi et al., 2020; Kalckert \& Ehrsson, 2012, 2014; 
Pyasik et al., 2018). They are known to rely at least partially on different processes, where ownership is mostly dependent on the direct integration of multisensory signals, while the sense of agency requires both predictive and inferential processes to interpret causality of self-generated actions (Seghezzi et al., 2019). Agency can more readily be experienced for external objects and tools, and thus might be less dependent on visual resemblance with one's own body than ownership (Maravita \& Iriki, 2004).

Furthermore, children enjoyed the experience more than adults, which might bear potential for VR-based applications. Crucially, children and adults demonstrated comparably low levels of suggestibility as measured by a control statement, thus suggesting that these observed differences were related to experimental manipulations rather than differences in answering styles between children and adults.

\section{Sense of Body Ownership}

The feeling that our body belongs to ourselves has been discussed as a fundamental aspect of the bodily self (Blanke, 2012). Using multisensory stimulation paradigms, such as the rubber hand or the full body illusion, it has been shown that adults generally experience more ownership of an artificial limb or body when the multisensory cues on the physical and artificial body are temporally synchronous and spatially coherent. Research in children demonstrated that 4-5-year-old children reported generally high levels of ownership both after synchronous and asynchronous visuotactile stimulation, while 10-13-year-old children responded more like adults, with higher levels of ownership after synchronous, than asynchronous stimulation (Cowie et al., 2013, 2016). Children's proprioceptive drift responses only became adult-like from around 10 years of age (Cowie et al., 2013, 2016). Similar findings were reported in a non-visual version of the rubber hand illusion, where 9year-old children demonstrated drift towards the rubber hand after synchronous, but not asynchronous stimulation, similar to adults, but 5-year-old children did not (Nava et al., 
2017). We did not observe differences between the two age groups in our data and the expected effects of synchrony and avatar type were shown in the explicit measures, both for adults and children. The children tested in this study were between 8 and 12 years old, a period that has been discussed in previous studies to be important for the development of the bodily self (Cowie et al., 2016). A study that used a third-person perspective full body illusion suggests that around this age specifically, children might transition from body perception that is primarily driven by visual information, to a more adult-like integration of multiple senses (Cowie et al., 2017). The study demonstrated that the integration of synchronous visual and tactile cues constitutes self-identification with the body seen from a third-person perspective in all tested age groups. Asynchronous visuotactile also cues led to self-identification in the 6-7-year-old children, which decreased in 8-11-year-old children, and even more so in young adults. Is suggests that the visual signals drive self-identification in younger children, whereas the integration of visual and tactile signals becomes increasingly important up to young adulthood. It is likely that such maturation translates to a body that is observed from a first-person perspective, as similar results were found for the rubber hand illusion. Taking together the results from the current study and those from a recent preprint (Keenaghan, Polaskova, et al., 2020), a similar maturation might occur for the integration of visuomotor as compared to visuotactile signals. The preprint described that in a similar first-person perspective full body illusion as we used here, 5-year-old children reported a strong feeling of ownership both after synchronous and asynchronous visuomotor stimulation (Keenaghan, Polaskova, et al., 2020). In contrast to the findings by Keenaghan and colleagues (2020), the children in our sample showed an adult-like modulation of synchrony on ownership, confirming the idea that other sensory signals, in addition to the visual first-person perspective become increasingly important at that age. Noteworthy is that also in adults, the mere sight of an artificial body(part) from a natural first-person perspective 
might induce a feeling of ownership for this body(part) (Carey et al., 2019), but that incongruent multisensory information breaks the illusory feeling of ownership (Keenaghan, Bowles, et al., 2020). This effect of asynchronous multisensory information might become increasingly pronounced across childhood, which could be a potential explanation for why we observed similar effects as in adults in 8-12-year-old children, but Keenaghan and colleagues (2020) did not in 5-year-old children.

The SCR to bodily threat, a common implicit measure of body ownership, has previously shown to be stronger after synchronous than asynchronous stimulation in the rubber hand illusion and first-person perspective video-based full body illusions (i.e. Armel \& Ramachandran, 2003; Petkova \& Ehrsson, 2008; Petkova et al., 2011; Preuss \& Ehrsson, 2019; Tacikowski et al., 2020). However, while we could evidence threat response in general, these responses were not modulated by experimental condition, which is in line with other studies using virtual avatars (Kokkinara \& Slater, 2014; Slater et al., 2010). A potential explanation for these diverging findings might be related to the online head-tracking typically used in virtual reality but not in video-based settings, which has been suggested to strongly enhance the experience of embodiment (Maselli \& Slater, 2013; Roel Lesur et al., 2018). Another consideration is that the threat used in the current study (i.e. the sight of an object falling from the ceiling), might have been arousing on a more general level, which was not specific enough to directly threaten the integrity of the body (Braithwaite et al., 2020). Thus, the SCR here might reflect a generally arousing effect, independent of the level of ownership for the virtual avatar. The threat used in this study was chosen to not be too traumatizing for the children, but still threatening. Future studies might aim to find appropriate threats for children, to support findings on subjective experiences of embodiment with physiological measures. 


\section{Sense of Agency}

The sense of being in control over one's own bodily actions and their consequences in the environment has been described as another core aspect of embodiment (Gallagher, 2000). While certain aspects of agency have been shown to change across development, such as the learning of causal relations between self-generated actions and their outcomes in infancy (Zaadnoordijk et al., 2020), or metacognitive awareness in childhood (Metcalfe et al., 2010), the explicit sense of bodily agency has rarely been investigated in children. Here, for the sense agency of a full body in VR, we found that adults experienced less agency for the skeleton than the human avatar. On the other hand, human likeness of the avatar did not modulate the experience of agency in children, which might suggest that the top-down expectancies of what an avatar should look like for it to be embodied are more flexible in children than in adults.

Research with adults shows that the feeling of agency for virtual avatars is strongly reduced with visuomotor asynchrony (Keenaghan, Bowles, et al., 2020). In accordance, our data in adults showed a reduced sense of agency with increasing visuomotor delay. Children, on the other hand, showed a generally enhanced subjective sense of agency compared to adults, even when there are larger mismatches between the self-performed movements and the visual feedback about the avatar's movements. Even though agency was generally reduced for asynchronous conditions as compared to the synchronous condition in children, it did not reduce with increasing delays, like it did for adults. Moreover, we also did not observe a difference in agency between the two children's age groups in exploratory analyses. The difference in the experience of agency that we observed between children and adults might reflect that that the ongoing matching between the motor intention and predicted and actual sensory outcomes might still be under development in children. This is in line with the limited evidence in children which demonstrates that visuomotor synchrony is not a 
prerequisite for the experience of agency in five-year-old children (Keenaghan, Polaskova, et al., 2020). Taken together, these findings might reflect a developmental trajectory in the experience of agency for virtual avatars, which is different for the experience of ownership. In other contexts, it is shown that children's judgements of agency are not fully adultlike (Metcalfe et al., 2010), and that they might exhibit a self-attribution bias, such that they are more likely to report being the agent of an action when the outcome is positive (van Elk et al., 2015). It has been argued, that these explicit agency judgements would require a sense of responsibility for the action outcome, which involves the frontal lobes that are still developing during late childhood (Cavazzana et al., 2014). While we did not measure task performance in the cube-task, successful interactions with the cubes (i.e. the cubes changing colors) even in case of asynchrony could potentially drive the high levels of agency in the asynchronous conditions in children.

Both synchrony detection between motor intents and sensory outcomes, and between different multisensory inputs have been discussed as crucial factors underlying of the sense of self in infant literature, showing that a basic sense of the body might be present from very early on in development (Rochat, 2003). However, this process likely undergoes fine-tuning throughout childhood, facilitating the maintenance of a stable sense of bodily self even though the physical body itself is rapidly changing. Overall, our data demonstrated adult-like modulations in ownership were found in the children's data, but the assessment of agency provided a different pattern of results, whereas agency and ownership were affected in similar ways by our experimental manipulations for adults. In children, agency was generally higher in children, not affected by visual appearance of the avatar, and not different between the two asynchronous conditions. This corroborates recent evidence that even though agency and ownership are closely related, they might depend on different mechanisms that do not develop at the same rate. More specifically, visual capture might drive the feeling of agency 
in children up to at least 12 years-old, whereas the integration of visual and sensorimotor information drives the feeling of ownership. Yet, future studies will have to more carefully investigate and contrast the influence of various multisensory and sensorimotor contingencies on the sense of ownership and agency across childhood, ideally employing both explicit and implicit measures.

\section{Limitations and outlook}

This study aimed to provide evidence from both subjective reports and physiological correlates for the embodiment of virtual avatars in children and adults. While children did not differ from adults in the control question, it is known that subjective measures of bodily illusions can strongly vary between adult participants, and children expectedly show an even more heterogenous response. Even if we used a rather conservative non-parametric approach to account for that, we cannot exclude that it affected power. In particular, the exploratory analyses within the children group should be interpreted with caution due to the limited sample size. As a consequence, the subjective reports might thus be somewhat limited, and the use of additional objective and implicit measures are important. In line with previous studies, we used SCR to a threat as an implicit measure of ownership, but as discussed, we did not observe a difference between experimental conditions. Interestingly, we observed an interaction of age group and repetition, demonstrating that adults show the expected strong reduction in SCRs with repeated exposures to the threat (Dawson et al., 2000). In children, no such attenuation was observed and responses remained high also after repeated threats. Studies in different contexts suggest differential SCRs across development, that might strongly depend on aspects of the experimental design (Gao et al., 2010; Latzman et al., 2006). Future studies should address these developmental aspects of the SCR more in detail, and tailor the experimental design and threat stimuli so that it specifically reflects the experience of a bodily threat and not only general arousal levels in children. 
The current study is one of the first to test how children embody virtual avatars depending on visual appearance and visuomotor contingency. Our results showed that children can easily embody avatars, even if they do not look like themselves. Furthermore, they enjoyed the experience to a high-level, and even more than adults. This suggests embodied VR as a promising tool to both study embodied cognition and for various applications, but also shows potential caveats that should be addressed in future studies. We thus believe that this research is important, not only from a fundamental perspective, but also for the many possible implications in educational and clinical settings (Southgate et al., 2019; Won et al., 2017). 


\section{References}

Adolph, K. E., \& Hoch, J. E. (2019). Motor development: Embodied, embedded, enculturated, and enabling. Annual Review of Psychology, 70(1), 141-164. https://doi.org/10.1146/annurev-psych-010418-102836

Allen, M., Poggiali, D., Whitaker, K., Marshall, T. R., \& Kievit, R. A. (2019). Raincloud plots: A multi-platform tool for robust data visualization. Wellcome Open Research, 4, 63. https://doi.org/10.12688/wellcomeopenres.15191.1

Apps, M. A. J., \& Tsakiris, M. (2014). The free-energy self: A predictive coding account of self-recognition. Neuroscience and Biobehavioral Reviews, 41, 85-97. https://doi.org/10.1016/j.neubiorev.2013.01.029

Armel, K. C., \& Ramachandran, V. S. (2003). Projecting sensations to external objects: Evidence from skin conductance response. Proceedings of the Royal Society of London. Series B: Biological Sciences, 270(1523), 1499-1506. https://doi.org/10.1098/rspb.2003.2364

Bahrick, L. E. (2013). Body perception: Intersensory origins of self and other perception in newborns. Current Biology, 23(23), R1039-R1041. https://doi.org/10.1016/j.tcb.2013.07.003

Bahrick, L. E., \& Lickliter, R. (2000). Intersensory redundancy guides attentional selectivity and perceptual learning in infancy. Developmental Psychology, 36(2), 190-201. https://doi.org/10.1037/0012-1649.36.2.190

Bahrick, L. E., \& Watson, J. S. (1985). Detection of intermodal proprioceptive-visual contingency as a potential basis of self-perception in infancy. Developmental Psychology, 21(6), 963-973. https://doi.org/10.1037/0012-1649.21.6.963

Blanke, O. (2012). Multisensory brain mechanisms of bodily self-consciousness. Nature Reviews Neuroscience, 13(8), 556-571. https://doi.org/10.1038/nrn3292 
Botvinick, M., \& Cohen, J. (1998). Rubber hands “feel” touch that eyes can see. Nature, 391. https://doi.org/10.1038/35784

Braithwaite, J. J., Watson, D. G., \& Dewe, H. (2020). The Body-Threat Assessment Battery (BTAB): A new instrument for the quantification of threat-related autonomic affective responses induced via dynamic movie clips. International Journal of Psychophysiology, 155, 16-31. https://doi.org/10.1016/j.ijpsycho.2020.04.018

Burr, D., \& Gori, M. (2011). Multisensory integration develops late in humans. In M. M. Murray \& M. T. Wallace (Eds.), The Neural Bases of Multisensory Processes (pp. 345-362). CRC Press/Taylor \& Francis.

Carey, M., Crucianelli, L., Preston, C., \& Fotopoulou, A. (2019). The effect of visual capture towards subjective embodiment within the full body illusion. Scientific Reports, 9(1), 2889. https://doi.org/10.1038/s41598-019-39168-4

Cavazzana, A., Begliomini, C., \& Bisiacchi, P. S. (2014). Intentional binding effect in children: Insights from a new paradigm. Frontiers in Human Neuroscience, 8. https://doi.org/10.3389/fnhum.2014.00651

Cioffi, M. C., Hackett, J., \& Moore, J. W. (2020). Synchronous stimulation in the rubber hand illusion task boosts the subsequent sense of ownership on the vicarious agency task. Consciousness and Cognition, 80, 102904. https://doi.org/10.1016/j.concog.2020.102904

Cowie, D., Makin, T. R., \& Bremner, A. J. (2013). Children's responses to the rubber-hand illusion reveal dissociable pathways in body representation. Psychological Science, 24(5), 762-769.

Cowie, D., McKenna, A., Bremner, A. J., \& Aspell, J. E. (2017). The development of bodily self-consciousness: Changing responses to the Full Body Illusion in childhood. Developmental Science, 00:e12557. https://doi.org/10.1111/desc.12557 
Cowie, D., Sterling, S., \& Bremner, A. J. (2016). The development of multisensory body representation and awareness continues to 10 years of age: Evidence from the rubber hand illusion. Journal of Experimental Child Psychology, 142, 230-238. https://doi.org/10.1016/j.jecp.2015.10.003

Dawson, M. E., Schell, A. M., \& Filion, D. L. (2000). The electrodermal system. In J. Cacioppo, Tassinary L, \& Berntson G (Eds.), Handbook of Psychophysiology (2nd. 2007, pp. 200-223). https://doi.org/10.1016/j.ces.2007.04.037

Gallagher, S. (2000). Philosophical conceptions of the self: Implications for cognitive science. Trends in Cognitive Sciences, 4(1), 14-21. https://doi.org/10.1016/S13646613(99)01417-5

Gao, Y., Raine, A., Venables, P. H., Dawson, M. E., \& Mednick, S. A. (2010). The development of skin conductance fear conditioning in children from ages 3 to 8 years: Development of conditioning. Developmental Science, 13(1), 201-212. https://doi.org/10.1111/j.1467-7687.2009.00874.x

Haans, A., IJsselsteijn, W. A., \& de Kort, Y. A. W. (2008). The effect of similarities in skin texture and hand shape on perceived ownership of a fake limb. Body Image, 5(4), 389-394. https://doi.org/10.1016/j.bodyim.2008.04.003

Kalckert, A., \& Ehrsson, H. H. (2012). Moving a rubber hand that feels like your own: A dissociation of ownership and agency. Frontiers in Human Neuroscience, 6. https://doi.org/10.3389/fnhum.2012.00040

Kalckert, A., \& Ehrsson, H. H. (2014). The moving rubber hand illusion revisited: Comparing movements and visuotactile stimulation to induce illusory ownership. Consciousness and Cognition, 26, 117-132. https://doi.org/10.1016/j.concog.2014.02.003 
Kay, M., \& Wobbrock, J. (2020). ARTool: Aligned rank transform for nonparametric factorial ANOVAs (R package version 0.10.7) [Computer software]. https://github.com/mjskay/ARTool. 10.5281/zenodo.594511

Keenaghan, S., Bowles, L., Crawfurd, G., Thurlbeck, S., Kentridge, R. W., \& Cowie, D. (2020). My body until proven otherwise: Exploring the time course of the full body illusion. Consciousness and Cognition, 78, 102882. https://doi.org/10.1016/j.concog.2020.102882

Keenaghan, S., Polaskova, M., Thurlbeck, S., Kentridge, R. W., \& Cowie, D. (2020). Alice in Wonderland: The effects of body size and movement on children's size perception and bodily awareness in virtual reality [Preprint]. PsyArXiv. https://doi.org/10.31234/osf.io/uw768

King, B. R., Pangelinan, M. M., Kagerer, F. A., \& Clark, J. E. (2010). Improvements in proprioceptive functioning influence multisensory-motor integration in 7- to 13-yearold children. Neuroscience Letters, 483(1), 36-40. https://doi.org/10.1016/j.neulet.2010.07.056

Kokkinara, E., \& Slater, M. (2014). Measuring the effects through time of the influence of visuomotor and visuotactile synchronous stimulation on a virtual body ownership illusion. Perception, 43(1), 43-58. https://doi.org/10.1068/p7545

Latzman, R. D., Knutson, J. F., \& Fowles, D. C. (2006). Schedule-induced electrodermal responding in children. Psychophysiology, 43(6), 623-632. https://doi.org/10.1111/j.1469-8986.2006.00459.x

Lenggenhager, B., Tadi, T., Metzinger, T., \& Blanke, O. (2007). Video ergo sum: Manipulating bodily self-consciousness. Science, 317(5841), 1096-1099. https://doi.org/10.1126/science.1143439 
Longo, M. R., Schüür, F., Kammers, M. P. M., Tsakiris, M., \& Haggard, P. (2008). What is embodiment? A psychometric approach. Cognition, 107(3), 978-998. https://doi.org/10.1016/j.cognition.2007.12.004

Maravita, A., \& Iriki, A. (2004). Tools for the body (schema). Trends in Cognitive Sciences, 8(2), 79-86. https://doi.org/10.1016/j.tics.2003.12.008

Maselli, A., \& Slater, M. (2013). The building blocks of the full body ownership illusion. Frontiers in Human Neuroscience, 7. https://doi.org/10.3389/fnhum.2013.00083

Metcalfe, J., Eich, T. S., \& Castel, A. D. (2010). Metacognition of agency across the lifespan. Cognition, 116(2), 267-282. https://doi.org/10.1016/j.cognition.2010.05.009

Mills, K. L., Goddings, A.-L., Herting, M. M., Meuwese, R., Blakemore, S.-J., Crone, E. A., Dahl, R. E., Güroğlu, B., Raznahan, A., Sowell, E. R., \& Tamnes, C. K. (2016). Structural brain development between childhood and adulthood: Convergence across four longitudinal samples. NeuroImage, 141, 273-281. https://doi.org/10.1016/j.neuroimage.2016.07.044

Nardini, M., Begus, K., \& Mareschal, D. (2013). Multisensory uncertainty reduction for hand localization in children and adults. Journal of Experimental Psychology: Human Perception and Performance, 39(3), 773-787. https://doi.org/10.1037/a0030719

Nava, E., Bolognini, N., \& Turati, C. (2017). The development of a cross-modal sense of body ownership. Psychological Science, 28(3), 330-337. https://doi.org/10.1177/0956797616682464

Nava, E., Gamberini, C., Berardis, A., \& Bolognini, N. (2018). Action shapes the sense of body ownership across human development. Frontiers in Psychology, 9. https://doi.org/10.3389/fpsyg.2018.02507 
Petkova, V. I., Björnsdotter, M., Gentile, G., Jonsson, T., Li, T. Q., \& Ehrsson, H. H. (2011). From part- to whole-body ownership in the multisensory brain. Current Biology, 21(13), 1118-1122. https://doi.org/10.1016/j.cub.2011.05.022

Petkova, V. I., \& Ehrsson, H. H. (2008). If I were you: Perceptual illusion of body swapping. PLoS ONE, 3(12), e3832-e3832. https://doi.org/10.1371/journal.pone.0003832

Power, J. D., Fair, D. A., Schlaggar, B. L., \& Petersen, S. E. (2010). The development of human functional brain networks. Neuron, 67, 735-748. https://doi.org/10.1016/j.neuron.2010.08.017

Preuss, N., \& Ehrsson, H. H. (2019). Full-body ownership illusion elicited by visuovestibular integration. Journal of Experimental Psychology: Human Perception and Performance, 45(2), 209-223. https://doi.org/10.1037/xhp0000597

Pyasik, M., Burin, D., \& Pia, L. (2018). On the relation between body ownership and sense of agency: A link at the level of sensory-related signals. Acta Psychologica, 185, 219228. https://doi.org/10.1016/j.actpsy.2018.03.001

R Core Team. (2018). R: A language and environment for statistical computing. $R$ Foundation for Statistical Computing (3.5.1) [Computer software]. https://www.Rproject.org/

Riva, G. (2018). The neuroscience of body memory: From the self through the space to the others. Cortex, 104, 241-260. https://doi.org/10.1016/j.cortex.2017.07.013

Rochat, P. (2003). Five levels of self-awareness as they unfold early in life. Consciousness and Cognition, 12(4), 717-731. https://doi.org/10.1016/S1053-8100(03)00081-3

Roel Lesur, M., Aicher, H., Delplanque, S., \& Lenggenhager, B. (2020). Being short, sweet, and sour: Congruent visuo-olfactory stimulation enhances illusory embodiment. Perception, 49(6), 693-696. https://doi.org/10.1177/0301006620928669 
Roel Lesur, M., Gaebler, M., Bertrand, P., \& Lenggenhager, B. (2018). The plasticity of the bodily self: Head movements in bodily illusions and their relation to Gallagher's body image and body schema. Constructivist Foundations, 14(1), 94-105. https://constructivist.info/14/1/094

Roel Lesur, M., Weijs, M. L., Simon, C., Kannape, O. A., \& Lenggenhager, B. (2020). Psychometrics of disembodiment and its differential modulation by visuomotor and visuotactile mismatches. IScience, 23(3), 100901. https://doi.org/10.1016/j.isci.2020.100901

Seghezzi, S., Giannini, G., \& Zapparoli, L. (2019). Neurofunctional correlates of bodyownership and sense of agency: A meta-analytical account of self-consciousness. Cortex, 121, 169-178. https://doi.org/10.1016/j.cortex.2019.08.018

Slater, M., Spanlang, B., Sanchez-Vives, M. V., \& Blanke, O. (2010). First person experience of body transfer in virtual reality. PloS One, 5(5), e10564. https://doi.org/10.1371/journal.pone.0010564

Southgate, E., Smith, S. P., Cividino, C., Saxby, S., Kilham, J., Eather, G., Scevak, J., Summerville, D., Buchanan, R., \& Bergin, C. (2019). Embedding immersive virtual reality in classrooms: Ethical, organisational and educational lessons in bridging research and practice. International Journal of Child-Computer Interaction, 19, 1929. https://doi.org/10.1016/j.ijcci.2018.10.002

Spear, L. P. (2000). Neurobehavioral changes in adolescence. Current Directions in Psychological Science, 9(4), 111-114. https://doi.org/10.1111/1467-8721.00072

Tacikowski, P., Weijs, M. L., \& Ehrsson, H. H. (2020). Perception of our own body influences self-concept and self-incoherence impairs episodic memory. IScience, 101429. https://doi.org/10.1016/j.isci.2020.101429 
Thelen, E. (1992). Development as a dynamic system. Current Directions in Psychological Science, 1(6), 189-193. https://doi.org/10.1111/1467-8721.ep10770402

Tsakiris, M., \& Haggard, P. (2005). The rubber hand illusion revisited: Visuotactile integration and self-attribution. Journal of Experimental Psychology: Human Perception and Performance, 31(1), 80-91. https://doi.org/10.1037/00961523.31.1.80

Tsakiris, M., Prabhu, G., \& Haggard, P. (2006). Having a body versus moving your body: How agency structures body-ownership. Consciousness and Cognition, 15(2), 423432. https://doi.org/10.1016/j.concog.2005.09.004

van Elk, M., Rutjens, B. T., \& van der Pligt, J. (2015). The development of the illusion of control and sense of agency in 7- to-12-year old children and adults. Cognition, 145, 1-12. https://doi.org/10.1016/j.cognition.2015.08.004

Won, A., Bailey, J., Bailenson, J., Tataru, C., Yoon, I., \& Golianu, B. (2017). Immersive virtual reality for pediatric pain. Children, 4(7), 52. https://doi.org/10.3390/children4070052

Zaadnoordijk, L., Meyer, M., Zaharieva, M., Kemalasari, F., van Pelt, S., \& Hunnius, S. (2020). From movement to action: An EEG study into the emerging sense of agency in early infancy. Developmental Cognitive Neuroscience, 42, 100760. https://doi.org/10.1016/j.den.2020.100760

Zmyj, N., Jank, J., Schütz-Bosbach, S., \& Daum, M. M. (2011). Detection of visual-tactile contingency in the first year after birth. Cognition, 120(1), 82-89. https://doi.org/10.1016/j.cognition.2011.03.001 


\section{Table 1}

Embodiment Questionnaire

\begin{tabular}{lll}
\hline & Category & Statement \\
\hline 1 & ownership & I felt as if the seen body was my body \\
2 & agency & I felt like I could move the seen body as I wanted to \\
3 & threat & It felt as if the falling brick could have actually hurt me \\
4 & strangeness & I felt strange in the seen body \\
5 & emotional experience & I enjoyed the experience \\
6 & control statement & It felt as if the color of the seen body changed to green \\
\hline
\end{tabular}




\section{Figure 1}

Experimental Procedure and Setup

A.

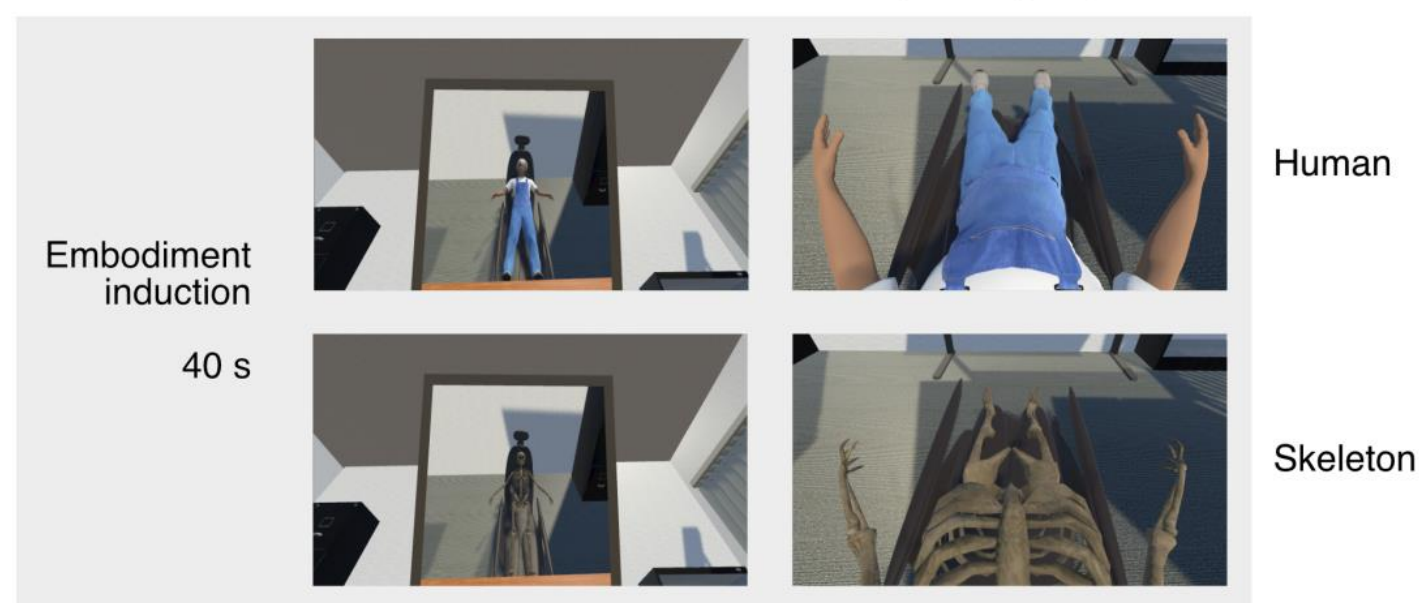

B.

$$
\begin{array}{r}
\text { Interactive } \\
\text { game }
\end{array}
$$

$90 \mathrm{~s}$
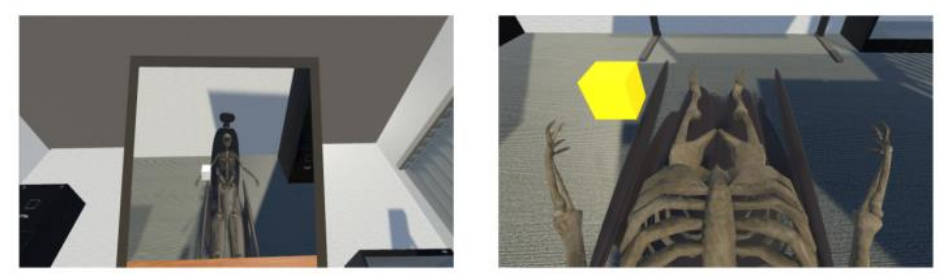

c.
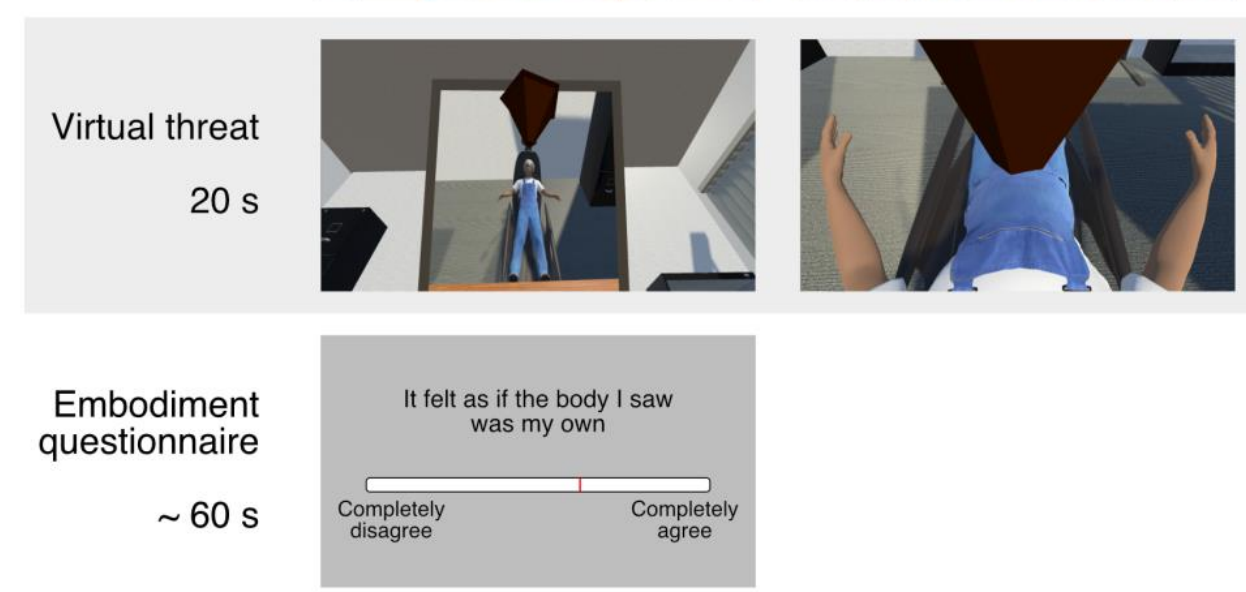

D.

Note. Views of the avatar are presented from both a mirror perspective, and a first-person perspective. Within one condition, the same avatar was presented during the embodiment induction, interactive game, and virtual threat. A) During the embodiment induction, participants moved around and observed the avatar from both the first-person perspective and mirror perspective. B) In the interactive game, participants could freely switch between the two perspective. White cubes appeared, which turned yellow after a successful interaction. C) 
The virtual threat was a diamond-shaped object that slowly fell from the ceiling and moved through the avatar's body. It could be first seen in the mirror-perspective, and only later in the first-person perspective, participants could freely switch between the two perspectives. D) Finally, an embodiment questionnaire, consisting of six statements, was shown on the HMD, which participants could answer with their head movements. 


\section{Figure 2}

Responses to the Ownership and Agency Statement

Adults

A. Ownership
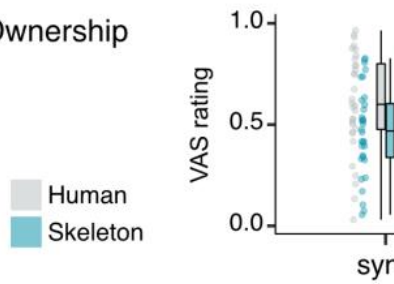

B. Agency

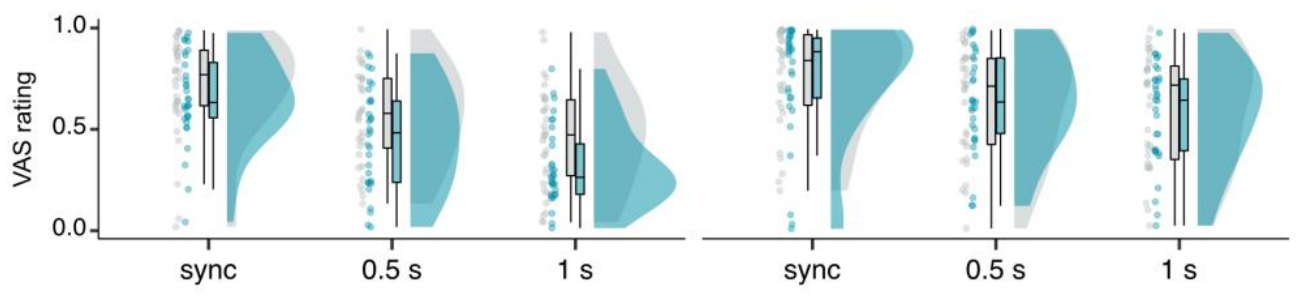

Note. Responses to the A) ownership statement and B) agency statement of the embodiment questionnaire in all experimental conditions (synchronous, $0.5 \mathrm{~s}$ delay, $1 \mathrm{~s}$ delay), plotted separately for adults and children. Colors indicate the different avatars. Dots indicate the individual responses in each condition, boxes indicate the interquartile range, with horizontal lines marking the median, and whiskers indicate the lower and upper extreme. 


\section{Figure 3}

Objective and Subjective Responses to the Virtual Threat

Adults

A. Subjective

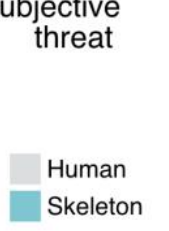

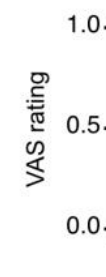

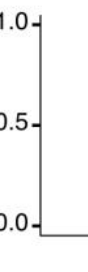

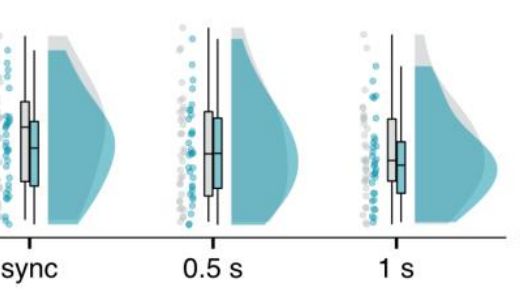

B. Objective

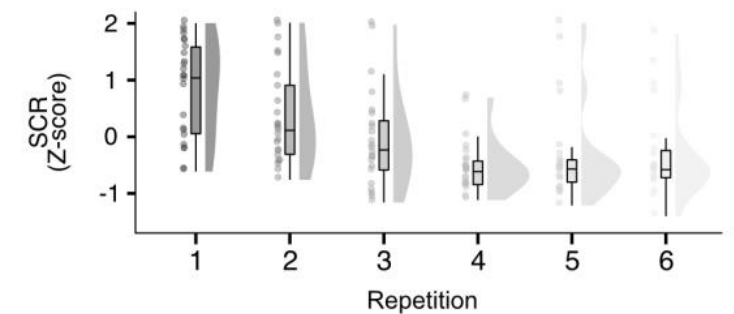

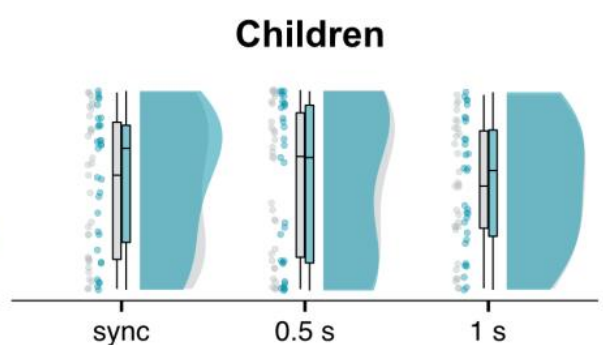

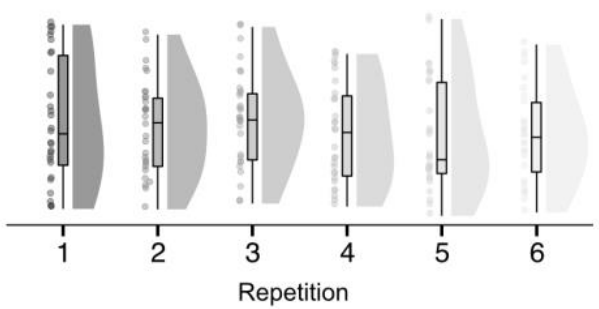

Note. A) Agreement with the statement: 'It felt as if the falling brick could have actually hurt me' (i.e. subjective threat) plotted by condition (synchronous, $0.5 \mathrm{~s}$ delay, $1 \mathrm{~s}$ delay), plotted separately for adults and children. Colors indicate the different avatars. B) Z-scored SCRs (i.e. objective threat) plotted for the significant interaction of group and repetition, independently of experimental condition. Dots indicate the individual responses in each condition, boxes indicate the interquartile range, with horizontal lines marking the median, and whiskers indicate the lower and upper extreme. 